\title{
Some Result on Contact Pseudo-Slant Submanifolds of a Sasakian Manifold
}

\author{
Süleyman DİRIKK \\ Department of Statistics, University of Amasya, 05250, Amasya, TURKEY \\ suleyman.dirik@amasya.edu.tr
}

\begin{abstract}
In this paper, we study the geometry of the contact pseudo-slant submanifolds of a Sasakian manifold. We derive the integrability conditions of distributions in the definition of a contact pseudo-slant submanifold. The notions contact pseudo-slant product is defined, and the necessary and sufficient conditions for a submanifold to contact pseudo-slant product is given. Also, a non-trivial example is used to demonstrate that the method presented in this paper is effective.
\end{abstract}

Keywords: Sasakian Manifold, Slant Submanifold, Contact Pseudo-Slant Submanifold, Contact Pseudo-Slant Product.

\section{Introduction}

The differential geometry of slant submanifolds has shown an increasing development since B. Y. Chen defined slant submanifolds in complex manifolds as a natural generalization of both the invariant and anti-invariant submanifolds[5]. After then, Papaghuic initiated the notion of semi-slant submanifolds as a generalization of slant submanifolds and CR-submanifolds[12]. Furthermore, Carriazo defined pseudo-slant submanifold with the name anti-slant submanifolds as a special class of bi-slant submanifolds [2, 3, 4]. Also, pseudo-slant submanifolds have been studied by Khan et. al. in [10]. Later, U. C. De et. al. studied and characterized pseudoslant submanifolds of trans-Sasakian Manifolds [6]. Recently, M. Atceken and S. Dirik also have investigated contact pseudo-slant submanifolds in Cosymplectic, Kenmotsu, and Sasakian space forms and gave some results on mixed-geodesic, totally geodesic and the induced tensor fields to be parallel $[7,8,9]$.

In this paper, we study geometry of the contact pseudo-slant submanifolds of a Sasakian manifold. In Section 2, we review basic formulas and definitions for a Sasakian manifold and their submanifolds. In Section 3, we derive the integrability conditions of distributions in the definition of a contact pseudo-slant submanifold. The notions contact pseudo-slant product is defined, and the necessary and sufficient conditions for a submanifold to be contact pseudo-slant product is given. Also, a non-trivial example is used to demonstrate that the method presented in this paper is effective.

\section{Preliminaries}

Given an odd-dimensional Riemannian manifold $(\bar{M}, g)$, let $\varphi$ be a $(1,1)$-type tensor field $\xi$ is a unit vector field and $\eta$ is a 1 -form on $\bar{M}$. If we have

$\varphi^{2} X=-X+\eta(X) \xi, g(X, \xi)=\eta(X)$

and

$g(\varphi X, \varphi Y)=g(X, Y)-\eta(X) \eta(Y)$

for any vector fields $X, Y$ on $\bar{M}$, then $\bar{M}$ is said to have an almost contact metric structure $(\varphi, \xi, \eta, g)$ and it is called an almost contact metric manifold. 
Let $\Phi$ denote the fundamental form 2-form in $\bar{M}$, given by $\Phi(X, Y)=g(X, \varphi Y)$, for any vector fields $X, Y$ on $\bar{M}$. If $\Phi=d \eta$, then $\bar{M}$ is said to be a contact metric manifold. Furthermore, the contact metric structure is called a K-contact structure if $\xi$ is a Killing vector field, that is, $\bar{\nabla}_{X} \xi=-\varphi X$, for any vector field $X$ on $\bar{M}$ , where $\bar{\nabla}$ denotes the Levi-Civita connection on $\bar{M}$.

The structure $(\varphi, \xi, \eta, g)$ is said to be normal if $[\varphi, \varphi]+2 d \eta \otimes \xi=0$, where $[\varphi, \varphi]$ is the Nijenhuis torsion of $\varphi$. A normal contact metric manifold is called a Sasakian manifold. So every Sasakian manifold is a K-contact manifold. It is well-know that an almost contact metric manifold is a Sasakian if and only if

$$
\left(\bar{\nabla}_{X} \varphi\right) Y=g(X, Y) \xi-\eta(Y) X
$$

for any vector fields $X, Y$ on $\bar{M}$.

Let $\bar{M}(c)$ be a Sasakian space form with constant $\varphi$-holomorphic sectional curvature $c$. Then the curvature tensor $\bar{R}$ of $\bar{M}(c)$ is given by

$$
\begin{aligned}
\bar{R}(X, Y) Z= & \left(\frac{c+3}{4}\right)\{g(Y, Z) X-g(X, Z) Y\}+\left(\frac{c-1}{4}\right)\{\eta(X) \eta(Z) Y-\eta(Y) \eta(Z) X \\
& +g(X, Z) \eta(Y) \xi-g(Y, Z) \eta(X) \xi+g(X, \varphi Z) \varphi Y-g(Y, \varphi Z) \varphi X+2 g(X, \varphi Y) \varphi Z\}
\end{aligned}
$$

for any vector fields $X, Y, Z$ on $\bar{M}(c)$.

Now, let $M$ be a submanifold of an almost contact metric manifold $\bar{M}$, we denote the induced connections on $M$ and the normal bundle $T^{\perp} M$ by $\nabla$ and $\nabla^{\perp}$, respectively, then the Gauss and Weingarten formulas are given by

$\bar{\nabla}_{X} Y=\nabla_{X} Y+h(X, Y)$

and

$\bar{\nabla}_{X} V=-A_{V} X+\nabla_{X}^{\perp} V$

for any $X, Y \in \Gamma(T M), V \in \Gamma\left(T^{\perp} M\right)$, where $h$ is the second fundamental form and $A_{V}$ is the Weingarten map associated with $V$ as

$$
g\left(A_{V} X, Y\right)=g(h(X, Y), V) .
$$

We denote the Riemannian curvature tensor of $M$ by $R$, then the Gauss equation imply

$$
\bar{R}(X, Y) Z=R(X, Y) Z-A_{h(Y, Z)} X+A_{h(X, Z)} Y+\left(\nabla_{X} h\right)(Y, Z)-\left(\nabla_{Y} h\right)(X, Z),
$$

for any $X, Y, Z \in \Gamma(T M)$.

The covariant derivative $\nabla h$ of $h$ is defined by 
$\left(\nabla_{X} h\right)(Y, Z)=\nabla_{X}^{\perp} h(Z, Y)-h\left(\nabla_{X} Y, Z\right)-h\left(\nabla_{X} Z, Y\right)$,

and the covariant derivative $\nabla A$ of $A$ is also defined by

$\left(\nabla_{X} A\right)_{V} Y=\nabla_{X}\left(A_{V} Y\right)-A_{\nabla_{X} \frac{1}{V}} Y-A_{V} \nabla_{X} Y$

for any $X, Y \in \Gamma(T M)$ and $V \in \Gamma\left(T^{\perp} M\right)$.

Taking the normal component of (2.8), we reach at equation of Codazzi

$(\bar{R}(X, Y) Z)^{\perp}=\left(\nabla_{X} h\right)(Y, Z)-\left(\nabla_{Y} h\right)(X, Z)$.

If $(\bar{R}(X, Y) Z)^{\perp}=0$, then submanifold is said to be curvature-invariant.

The Ricci equation is given by

$g\left(R^{\perp}(X, Y) V, U\right)=g(\bar{R}(X, Y) U, V)-g\left(\left[A_{V}, A_{U}\right] X, Y\right)$,

for any $X, Y \in \Gamma(T M)$ and $U, V \in \Gamma\left(T^{\perp} M\right)$. If $R^{\perp}=0$, then the normal connection of the $M$ is called flat.

Furthermore, for any $X \in \Gamma(T M)$, we can write

$\varphi X=T X+N X$

where $T X$ and $N X$ denote the tangential and normal components of $\varphi X$, respectively. Similarly, for $V \in \Gamma\left(T^{\perp} M\right), \varphi V$ also can be written

$\varphi V=B V+C V$,

where $B V$ and $C V$ denote, respectively, the tangential and normal components of $\varphi V$.

Taking into account (2.4) and (2.12), we have

$$
\begin{aligned}
g\left(\bar{R}^{\perp}(X, Y) V, U\right)= & g\left(\left[A_{V}, A_{U}\right] X, Y\right)+\left(\frac{c-1}{4}\right)\{g(X, \varphi V) g(U, \varphi Y) \\
& -g(Y, \varphi V) g(\varphi X, U)+2 g(X, \varphi Y) g(\varphi V, U)\},
\end{aligned}
$$

for any $X, Y \in \Gamma(T M)$ and $V, U \in \Gamma\left(T^{\perp} M\right)$.

By using (2.4) and (2.8), the Riemannian curvature tensor $R$ of an immersed submanifold $M$ of a Sasakian space form $\bar{M}(c)$ is given by

$R(X, Y) Z=\left(\frac{c+3}{4}\right)\{g(Y, Z) X-g(X, Z) Y\}+\left(\frac{c-1}{4}\right)\{\eta(X) \eta(Z) Y-\eta(Y) \eta(Z) X$ 


$$
\begin{aligned}
& +\eta(Y) g(X, Z) \xi-\eta(X) g(Y, Z) \xi+g(X, \varphi Z) \varphi Y-g(Y, \varphi Z) \varphi X+2 g(X, \varphi Y) \varphi Z\} \\
& +A_{h(Y, Z)} X-A_{h(X, Z)} Y+\left(\nabla_{Y} h\right)(X, Z)-\left(\nabla_{X} h\right)(Y, Z) .
\end{aligned}
$$

The normal part of (2.16), we have

$$
\left(\nabla_{X} h\right)(Y, Z)-\left(\nabla_{Y} h\right)(X, Z)=\left(\frac{c-1}{4}\right)\{g(X, T Z) N Y-g(Y, T Z) N X+2 g(X, T Y) N Z\}
$$

By using (2.1), (2.13), (2.14) and taking into account of $\xi$ being tangent to $M$, we get

$$
T^{2}+B N=-I+\eta \oplus \xi, N T+C N=0
$$

and

$$
T B+B C=0, N B+C^{2}=-I .
$$

Furthermore, the covariant derivatives of the tensor field $T, N, B$ and $C$ are, respectively, defined by

$\left(\nabla_{X} T\right) Y=\nabla_{X} T Y-T \nabla_{X} Y$

$\left(\nabla_{X} N\right) Y=\nabla_{X}^{\perp} N Y-N \nabla_{X} Y$

$\left(\nabla_{X} B\right) V=\nabla_{X} B V-B \nabla_{X}^{\perp} V$

and

$\left(\nabla_{X} C\right) V=\nabla_{X}^{\perp} C V-C \nabla_{X}^{\perp} V$

for any $X, Y \in \Gamma(T M)$ and $V \in \Gamma\left(T^{\perp} M\right)$.

By using (2.4), (2.5), (2.6) and (2.13), we can easily to see that

$$
\begin{aligned}
& \left(\nabla_{X} T\right) Y=A_{N Y} X+B h(X, Y)+g(X, Y) \xi-\eta(Y) X, \\
& \left(\nabla_{X} N\right) Y=-h(X, T Y)+C h(X, Y), \\
& \left(\nabla_{X} B\right) V=A_{C V} X-T A_{V} X, \\
& \left(\nabla_{X} C\right) V=-N A_{V} X-h(X, B V)
\end{aligned}
$$

and for any $X, Y \in \Gamma(T M)$ and $V \in \Gamma\left(T^{\perp} M\right)$.

By using (2.25) and (2.26), we can easily to see that $g\left(\left(\nabla_{X} N\right) Y, V\right)=-g(h(X, T Y), V)+g(C h(X, Y), V)$ 


$$
\begin{aligned}
& =-g\left(A_{V} X, T Y\right)-g(h(X, Y), C V) \\
& =g\left(T A_{V} X, Y\right)-g\left(A_{C V} X, Y\right) \\
& =g\left(T A_{V} X-A_{C V} X, Y\right) \\
& =-g\left(A_{C V} X-T A_{V} X, Y\right)=-g\left(\left(\nabla_{X} B\right) V, Y\right)
\end{aligned}
$$

thus we have

$g\left(\left(\nabla_{X} N\right) Y, V\right)=-g\left(\left(\nabla_{X} B\right) V, Y\right)$.

Since $\xi$ is tangent to $M$, making use of (2.5), (2.6), (2.7) and (2.13), we infer that

$\nabla_{X} \xi=-T X, h(X, \xi)=-N X, A_{V} \xi=B V$

for all $V \in \Gamma\left(T^{\perp} M\right)$ and $X \in \Gamma(T M)$.

Definition 2.1 Let $M$ be a submanifold of an almost contact metric manifold $(\bar{M}, \varphi, \xi, \eta, g)$. Then $M$ is said to be a contact slant submanifold if the angle $\theta(X)$ between $\varphi X$ and $T_{M}(p)$ is constant at any point $p \in M$ for any $X$ linearly independent of $\xi$. Thus the invariant and anti-invariant submanifolds are special class of slant submanifolds with slant angles $\theta=0$ and $\theta=\frac{\pi}{2}$, respectively. If the slant angle $\theta$ is neither zero nor $\frac{\pi}{2}$, then slant submanifold is said to be proper contact slant submanifold. The slant submanifolds of an almost contact metric manifold, the following theorem is well known.[11]

Theorem 2.2 Let $M$ be a submanifold of an almost contact metric manifold $\bar{M}$ such that $\xi \in \Gamma(T M) . M$ is a contact slant submanifold if and only if there exists a constant $\lambda \in(0,1)$ such that

$T^{2}=\lambda(-I+\eta \otimes \xi)$

Furthermore, if $\theta$ is slant angle of $M$, then it satisfies $\lambda=\cos ^{2} \theta$ [3].

As a consequence of the above Theorem and (2.18), we have the following relations;

$$
\begin{aligned}
& g(T X, T Y)=\cos ^{2} \theta\{g(X, Y)-\eta(X) \eta(Y)\}, \\
& g(N X, N Y)=\sin ^{2} \theta\{g(X, Y)-\eta(X) \eta(Y)\}, \\
& B N=\sin ^{2} \theta(-I+\eta \oplus \xi) .
\end{aligned}
$$

For a slant submanifold $M$ of an almost contact metric manifold $\bar{M}$, the normal bundle $T^{\perp} M$ of $M$ is decomposable as

$T^{\perp} M=N(T M) \oplus \mu$,

where $\mu$ is the invariant normal subbundle with respect to $\varphi$. 


\section{Some Result on Contact Pseudo-Slant Submanifolds of a Sasakian Manifold}

In this section, we study contact pseudo-slant submanifolds in a Sasakian manifold and we give some characterization results.

Definition 3.1 Let $M$ be a submanifold of a Sasakian manifold $\bar{M}(\varphi, \xi, \eta, g)$. We say that $M$ is a contact pseudo-slant submanifold if there exists a pair of orthogonal distributions $D^{\theta}$ and $D^{\perp}$ on $M$ such that

i.) the distribution $D^{\perp}$ is anti-invariant, i.e., $\varphi\left(D^{\perp}\right) \subseteq T^{\perp} M$,

ii.) the distribution $D^{\theta}$ is slant with slant angle $\theta$,

iii.) the tangent space $T M$ admits the orthogonal direct decomposition

$$
T M=D^{\perp} \oplus D^{\theta}, \xi \in \Gamma(T M)[10] .
$$

If we denote the dimensions of $D^{\perp}$ and $D^{\theta}$ by $n$ and $m$, respectively, the we have the following possible cases;

i.) if $n=0$, then $M$ is a slant submanifold,

ii.) if $m=0$, then $M$ is an anti-invariant submanifold,

iii.) if $n m \neq 0, \theta=0$, then $M$ is a contact CR-submanifold.

For a pseudo-slant submanifold $M$ of a Sasakian manifold $\bar{M}$, the normal bundle $T^{\perp} M$ of a pseudo-slant submanifold $M$ is decomposable as

$$
T^{\perp} M=\varphi\left(D^{\perp}\right) \oplus N\left(D^{\theta}\right) \oplus \mu, \varphi\left(D^{\perp}\right) \perp N\left(D^{\theta}\right) .
$$

Theorem 3.2 Let $M$ be a contact pseudo-slant submanifold of a Sasakian manifold $\bar{M}$. Then anti-invariant distribution $D^{\perp}$ is always integrable.

Proof For any $Y, Z \in \Gamma\left(D^{\perp}\right)$, we have

$$
\begin{gathered}
\bar{\nabla}_{Z} \varphi Y=\varphi \bar{\nabla}_{Z} Y+g(Z, Y) \xi \\
-A_{N Y} Z+\nabla_{Z}^{\perp} N Y=T \nabla_{Z} Y+N \nabla_{Z} Y+B h(Z, Y)+C h(Z, Y)+g(Z, Y) \xi
\end{gathered}
$$

which implies that

$$
-A_{N Y} Z=T \nabla_{Z} Y+B h(Y, Z)+g(Z, Y) \xi
$$

Thus we have

$$
T[Y, Z]=A_{N Z} Y-A_{N Y} Z \text {. }
$$


Since the ambient manifold $\bar{M}$ is Sasakian, we have

$$
\begin{aligned}
g\left(A_{N Z} Y-A_{N Y} Z, U\right)= & g(h(Y, U), N Z)-g(h(Z, U), N Y) \\
= & g(h(Y, U), N Z)-g\left(\bar{\nabla}_{U} Z, N Y\right) \\
= & g(h(Y, U), N Z)+g\left(\bar{\nabla}_{U} \varphi Y, Z\right) \\
= & g(h(Y, U), N Z)+g\left(\left(\bar{\nabla}_{U} \varphi\right) Y+\varphi \bar{\nabla}_{U} Z, Z\right) \\
= & g(h(Y, U), N Z)+g\left(\varphi \bar{\nabla}_{U} Z, Z\right) \\
& +g(g(U, Y) \xi-\eta(Y) U, Z) \\
= & g(h(Y, U), N Z)-g\left(\bar{\nabla}_{U} Y, \varphi Z\right) \\
= & g(h(Y, U), N Z)-g(h(U, Y), N Z)=0,
\end{aligned}
$$

for any $U \in \Gamma(T M)$, that is,

$A_{N Z} Y=A_{N Y} Z$

From (3.2) and (3.3), we conclude that $T[Y, Z]=0$ i.e., $[Y, Z] \in \Gamma\left(D^{\perp}\right)$. The proof is completes.

Theorem 3.3 Let $M$ be contact pseudo-slant submanifold of a Sasakian manifold $\bar{M}$. Then the slant distribution $D^{\theta}$ is integrable if and only if

$g\left(A_{C N Y} Z+T A_{N Z} Y, X\right)=g\left(A_{C N X} Z+T A_{N Z} X, Y\right)$,

for any $X, Y \in \Gamma\left(D^{\theta}\right)$ and $Z \in \Gamma\left(D^{\perp}\right)$.

Proof By using (2.2) and (2.3), we have

$$
\begin{aligned}
g([X, Y], Z)= & g\left(\nabla_{X} Y, Z\right)-g\left(\nabla_{Y} X, Z\right) \\
= & g\left(\bar{\nabla}_{Y} Z, X\right)-g\left(\bar{\nabla}_{X} Z, Y\right) \\
= & g\left(\varphi \bar{\nabla}_{Y} Z, \varphi X\right)-g\left(\varphi \bar{\nabla}_{X} Z, \varphi Y\right) \\
= & g\left(\bar{\nabla}_{Y} \varphi Z-\left(\bar{\nabla}_{Y} \varphi\right) Z, \varphi X\right)-g\left(\bar{\nabla}_{X} \varphi Z-\left(\bar{\nabla}_{X} \varphi\right) Z, \varphi Y\right) \\
= & g\left(\bar{\nabla}_{Y} \varphi Z, \varphi X\right)-g(g(Y . Z) \xi+\eta(Z) Y, \varphi X) \\
& -g\left(\bar{\nabla}_{X} \varphi Z, \varphi Y\right)+g(g(X . Z) \xi+\eta(Z) X, \varphi Y) .
\end{aligned}
$$

Thus, from the equations numbered (2.5), (2.6), (2.7), (2.13) and (2.33) 


$$
\begin{aligned}
g([X, Y], Z)= & g\left(\bar{\nabla}_{Y} \varphi Z, T X\right)+g\left(\bar{\nabla}_{Y} \varphi Z, N X\right)-g\left(\bar{\nabla}_{X} \varphi Z, T Y\right)-g\left(\bar{\nabla}_{X} \varphi Z, N Y\right) \\
= & -g\left(A_{\varphi Z} T X, Y\right)+g\left(A_{\varphi Z} T Y, X\right)+g\left(\bar{\nabla}_{Z} X, \varphi N Y\right)-g\left(\bar{\nabla}_{Y} Z, \varphi N X\right) \\
= & g\left(A_{\varphi Z} T Y, X\right)-g\left(A_{\varphi Z} T X, Y\right)+g\left(\nabla_{X} Z, B N Y\right)-g\left(\nabla_{Y} Z, B N X\right) \\
& +g\left(\bar{\nabla}_{X} Z, C N Y\right)-g\left(\bar{\nabla}_{Y} Z, C N X\right) \\
= & g\left(A_{\varphi Z} T Y+T A_{\varphi Z} Y, X\right)-\sin ^{2} \theta g\left(\nabla_{X} Z, Y\right) \\
& +\sin ^{2} \theta g\left(\nabla_{Y} Z, X\right)+g(h(X, Z), C N Y)-g(h(Y, Z), C N X) \\
= & g\left(T A_{\varphi Z} Y+A_{\varphi Z} T Y, X\right)+\sin ^{2} \theta g([X, Y], Z)+g\left(A_{C N Y} X-A_{C N X} Y, Z\right),
\end{aligned}
$$

for any $X, Y \in \Gamma\left(D^{\theta}\right)$ and $Z \in \Gamma\left(D^{\perp}\right)$. Consequently, we reach at

$\cos ^{2} \theta g([X, Y], Z)=g\left(T A_{\varphi Z} Y+A_{\varphi Z} T Y, X\right)+g\left(A_{C N Y} X-A_{C N X} Y, Z\right)$,

which proves our assertion.

Definition 3.4 Let $M$ be a contact pseudo-slant submanifold of a Sasakian manifold $\bar{M} . M$ is said to be contact pseudo-slant product if the distributions $D^{\perp}$ and $D^{\theta}$ are totally geodesic in $M$.

Theorem 3.5 Let $M$ be a proper contact pseudo-slant submanifold of a Sasakian manifold $\bar{M}$. If the tensor field $N$ is parallel, then $M$ is a contact pseudo-slant product.

Proof $N$ is parallel if and only if $B$ is parallel, from (2.28) and (2.26), we have

$$
A_{C V} X-T A_{V} X=0
$$

taking $V=N Z$ in equation (3.4) and from (2.18), we get

$T A_{N Z} X=0, X \in \Gamma(T M), Z \in \Gamma\left(D^{\perp}\right)$.

This implies that $A_{\varphi Z} X \in \Gamma\left(D^{\perp}\right)$ and $B h(X, Z)=0$. The proof is completes.

Theorem 3.6 Let $M$ be a contact pseudo-slant submanifold of a Sasakian space form $\bar{M}(c)$. M is either anti-invariant submanifold or $\bar{M}$ is flat if $N$ is parallel.

Proof Since $N$ is parallel, we can easily to see that

$$
h(T X, Y)=C h(X, Y)=h(X, T Y),
$$

Thus we have

$$
g(h(T X, Y), V)=g(h(X, T Y), V)=g\left(A_{V} T X, Y\right)=g\left(A_{V} X, T Y\right)=-g\left(T A_{V} X, Y\right)
$$


which is equivalent to

$T A_{V} X+A_{V} T X=0$

for any $X, Y \in \Gamma(T M)$ and $V \in \Gamma\left(T^{\perp} M\right)$. From (3.5), we have

$$
g\left(A_{V} T X, B U\right)=g\left(A_{V} X, T B U\right)=-g\left(A_{V} X, B C U\right)=-g\left(N A_{V} X, C U\right)=0,
$$

for any vector fields $U, V$ normal to $M$. Taking the covariant derivative of (3.6), for $Y \in \Gamma(T M)$, we obtain

$$
g\left(\bar{\nabla}_{Y} A_{V} T X, B U\right)+g\left(A_{V} T X, \bar{\nabla}_{Y} B U\right)=0 .
$$

This means that

$0=g\left(\left(\nabla_{Y} A\right)_{V} T X+A_{\nabla_{Y}^{\perp} V} T X+A_{V} \nabla_{Y} T X, B U\right)+g\left(A_{V} T X,\left(\nabla_{Y} B\right) U+B \nabla_{Y}^{\perp} U\right)$.

Taking into account (2.28) and (3.6), we reach at

$$
g\left(\left(\nabla_{Y} A\right)_{V} T X+A_{V}\left\{\left(\nabla_{Y} T\right) X+T \nabla_{Y} X\right\}, B U\right)=0,
$$

from which

$$
g\left(\left(\nabla_{Y} A\right)_{V} T X, B U\right)+g\left(A_{V}\left\{A_{N X} Y+B h(X, Y)+g(X, Y) \xi-\eta(X) Y\right\}, B U\right)=0,
$$

or,

$$
g\left(\left(\nabla_{Y} A\right)_{V} T X, B U\right)+g\left(A_{V} B U, A_{N X} Y\right)+g\left(A_{V} B U, B h(X, Y)\right)=0 .
$$

This implies that

$$
g\left(\left(\nabla_{T Y} h\right)(T X, B U), V\right)=g\left(\left(\nabla_{T Y} A\right)_{V} T X, B U\right)=-g\left(A_{V} B U, A_{N X} T Y\right)-g\left(A_{V} B U, B h(T Y, X)\right) .
$$

Thus we conclude that

$$
\begin{aligned}
g\left(\left(\nabla_{T X} h\right)(T Y, B U)-\left(\nabla_{T Y} h\right)(T X, B U), V\right)= & g\left(A_{V} B U, A_{N X} T Y\right) \\
& -g\left(A_{V} B U, A_{N Y} T X\right) \\
& =g\left(A_{V} B U, A_{N X} T Y-T A_{N Y} X\right) \\
& =g\left(A_{V} A_{N X} T Y, B U\right) \\
& -g\left(A_{V} T A_{N Y} X, B U\right) \\
& =g\left(A_{V} T A_{N X} Y, B U\right)=0 .
\end{aligned}
$$

On the other hand, form the Codazzi equation, we have 


$$
\begin{aligned}
g\left(\left(\nabla_{T X} h\right)(T Y, B U)-\left(\nabla_{T Y} h\right)(T X, B U), V\right)= & \left(\frac{c-1}{4}\right)\left\{g\left(T^{2} Y, B V\right) g(N X, U)\right. \\
& -g\left(T^{2} X, B V\right) g(N Y, U) \\
& \left.+2 g\left(T X, T^{2} Y\right) g(N B V, U)\right\} \\
= & -\cos ^{2} \theta\left(\frac{c-1}{4}\right)\{g(Y, B V) g(N X, U) \\
& -g(X, B V) g(N Y, U) \\
& +2 g(T X, Y) g(N B V, U)\} .
\end{aligned}
$$

In (3.8), taking $X, Y \in \Gamma\left(D^{\theta}\right)$ and $U=V=N Z \in \Gamma\left(T^{\perp} M\right)$ for $Z \in \Gamma\left(D^{\perp}\right)$, and corresponding (3.7) and (3.8), we get

$\cos ^{2} \theta\left(\frac{c-1}{2}\right) g(T X, Y) g(Z, Z)=0$

This proves our assertion.

Example 3.9 Let $M$ be a submanifold of $\mathrm{R}^{9}$ defined by the following equation $\chi(u, v, w, t, z)=(3 u \sin \alpha,-v \cos \alpha,-2 u \sin \alpha, v \cos \alpha,-w \cos t, \cos t, w \sin t,-\sin t, z)$.

We can easily to see that the tangent bundle of $M$ is spanned by the tangent vectors $e_{1}=3 \sin \alpha \frac{\partial}{\partial x_{1}}-2 \sin \alpha \frac{\partial}{\partial x_{2}}, e_{2}=-\cos \alpha \frac{\partial}{\partial y_{1}}+\cos \alpha \frac{\partial}{\partial y_{2}}, e_{5}=\xi=\frac{\partial}{\partial z}$. $e_{3}=-\cos t \frac{\partial}{\partial x_{3}}+\sin t \frac{\partial}{\partial x_{4}}, e_{4}=w \sin t \frac{\partial}{\partial x_{3}}-\sin t \frac{\partial}{\partial y_{3}}+w \cos t \frac{\partial}{\partial x_{4}}-\cos t \frac{\partial}{\partial y_{4}}$.

For the almost contact metric structure $\varphi$ of $\mathrm{R}^{9}$, whose coordinate systems $\left(x_{1}, y_{1}, x_{2}, y_{2}, x_{3}, y_{3}, x_{4}\right.$, $\left.y_{4}, z\right)$, choosing $\varphi\left(\frac{\partial}{\partial x_{i}}\right)=\frac{\partial}{\partial y_{i}}, \varphi\left(\frac{\partial}{\partial y_{j}}\right)=-\frac{\partial}{\partial x_{j}}, 1 \leq i, j \leq 4$

then we have $\varphi e_{1}=3 \sin \alpha \frac{\partial}{\partial y_{1}}-2 \sin \alpha \frac{\partial}{\partial y_{2}}, \varphi e_{2}=\cos \alpha \frac{\partial}{\partial x_{1}}-\cos \alpha \frac{\partial}{\partial x_{2}}, \varphi e_{3}=-\cos t \frac{\partial}{\partial y_{3}}+\sin t \frac{\partial}{\partial y_{4}}$, and 
$\varphi e_{4}=w \sin t \frac{\partial}{\partial y_{3}}+\sin t \frac{\partial}{\partial x_{3}}+w \cos t \frac{\partial}{\partial y_{4}}+\cos t \frac{\partial}{\partial x_{4}}$.

By direct calculations, we can infer $D_{\theta}=\operatorname{span}\left\{e_{1}, e_{2}\right\}$ is a slant distribution with slant angle

$\cos \theta=\frac{g\left(e_{1}, \varphi e_{2}\right)}{\left\|e_{1}\right\|\left\|\varphi e_{2}\right\|}=\frac{5 \sin \theta \cdot \cos \theta}{\sqrt{13 \sin ^{2} \theta} \sqrt{2 \cos ^{2} \theta}}=\frac{5 \sqrt{26}}{26} \theta=\arccos \left(\frac{5 \sqrt{26}}{26}\right)$. Since $g\left(\varphi e_{3}, e_{i}\right)=0, \quad i=1,2,4,5$ and $\left(\varphi e_{4}, e_{j}\right)=0, j=1,2,3,5$ are orthogonal to $M, D^{\perp}=\operatorname{span}\left\{e_{3}, e_{4}, e_{5}\right\}$ is an anti-invariant

distribution. Thus $M$ is a 5 -dimensional proper contact pseudo-slant submanifold of $\mathrm{R}^{9}$ with it's usual almost contact metric structure.

\section{Conclusions and Remarks}

In this paper, we study geometry of the contact pseudo-slant submanifolds of a Sasakian manifold. We derive the integrability conditions of distributions in the definition of a contact pseudo-slant submanifold. The notions contact pseudo-slant product is defined and the necessary and sufficient conditions for a submanifold to be contact pseudo-slant product is given. Also, an non-trivial example is used to demonstrate that the method presented in this paper is effective. It is well known that open problems are so interesting in this area, especially for almost contact structures.

\section{Acknowledgments}

The author is grateful to the referee for valuable comments and suggestions

\section{References}

1. Blair D. E. and Showers D. K. (1974). Almost Contact Manifolds with Killing Structures II. J. Diff. Geom., 9:77582.

2. Carriazo A. (2000). Bi-Slant Immersions. In. Proc. ICARAMS 2000, Kharagpur, India, pp: 88-97.

3. Cabrerizo J. L., Carriazo A. L., Fernandez M. and Fernandez M. (2000). Slant submanifolds in Sasakian manifolds. Glasgow Math, J., 42:125-138.

4. Cabrerizo J. L., Carriazo, A. L. Fernandez, M, and Fernandez, M. (1999). Slant submanifolds in Sasakian manifolds. Geomeatriae Dedicata. 78:183-199.

5. Chen B-Y. (1990). Slant immersion. Bull. Austral. Math. Soc., 41: 135-147.

6. De U. C. and Sarkar. A. (2011). On Pseudo-Slant Submanifolds of Trans-Sasakian Manifolds, Proc. East Acad. Sci., 60: 1-11.

7. Dirik, S., and Atceken, M. (2016). Pseudo-Slant Submanifolds in Cosymplectic Space Forms. Acta Math. Sapientiae, 8(1): 53-74.

8. Dirik S., Atçeken M., and Yıldırım Ü. (2017). On pseudo-slant submanifolds of a Sasakian space form, Filomat, 31(19): 5909-5919.

9. Dirik S., Atçeken M., and Yıldııım Ü. (2016). Psudo Slant Submanifold in Kenmotsu Space Forms. Journal of Advances in Mathemaitics, 12(1): 5680-5696, 2016.

10. Khan V. A. and Khan M. A. (2007). Pseudo-Slant Submanifolds of Sasakian Manifold. Indian J. Pure and 
Appl. Math., 38: 31-42.

11. Lotta A. (1996). Slant submanifolds in contact geometry. Bulletin Mathematical Society Roumanie, 39: 183198.

12. Papaghuic N. (1994). Semi-Slant Submanifolds of a Kaehlerian Manifold. Ann. Şt. Al. I. Cuza Univ. Iaşi., 40: 55-61. 